\title{
DEVELOPMENT OF A NUMERICAL FRAMEWORK FOR VIRTUAL TESTING TO SUPPORT DESIGN OF A NEXT GENERATION THERMOPLASTIC MULTIFUNCTIONAL FUSELAGE
}

\author{
EMUS 2020 \\ B.H.A.H. TIJS ${ }^{* \dagger}$, K.S. VAN DOOREN ${ }^{\dagger}$ AND C. BISAGNI ${ }^{\dagger}$ \\ ${ }^{*}$ GKN Aerospace Fokker \\ Industrieweg 4, 3351 LB Papendrecht, The Netherlands \\ e-mail: bas.tijs@fokker.com,web page: https://www.gknaerospace.com/ \\ ${ }^{\dagger}$ Faculty of Aerospace Engineering, Aerospace Structures and Computational Mechanics \\ Delft University of Technology \\ Kluyverweg 1, 2629 HS Delft, The Netherlands \\ Web page: https://www.tudelft.nl/en/ae/
}

Key words: Numerical framework, Thermoplastic Composites, Virtual Testing, Welded Joints, Design

\begin{abstract}
This work summarizes the recent developments of a numerical framework to predict the mechanical behaviour of thermoplastic composites. It supports the design of a next generation thermoplastic multi-functional fuselage which uses advanced joining techniques such as thermoplastic welding to reduce both weight and cost by limiting the amount of mechanical fasteners required. At the lower end of the testing pyramid the framework is able to accurately predict typical preliminary design allowables such as laminate, open-hole and welded joints strength through a high-fidelity modelling approach. This information is then passed on to the structural level in a validated building-block approach to efficiently virtual test the compression strength of fuselage panels during post-buckling while also taking into account the influence of damages at the skin-stiffener interface.
\end{abstract}

\section{INTRODUCTION}

A numerical framework is currently in development to accurately predict matrix-dominated failure of thermoplastic composites and welded joints. It supports the design of a next generation thermoplastic multi-functional fuselage which uses advanced joining techniques such as thermoplastic welding to reduce both weight and cost by limiting the amount of mechanical fasteners required. However, using new thermoplastic composite materials and aiming for fastener-free joints also introduces new challenges. The strength of the highly loaded welded joints rely on the performance of the matrix and this matrix is strongly influenced by the manufacturing process. Furthermore, the state-of-the-art in modelling techniques is mostly developed and validated on thermoset composites, which show a more brittle failure behaviour [1].

The methodology to tackle these challenges is taking into account the constitutive mate-rial behavior of thermoplastic composites and accounts for the appropriate failure mechanisms 
through the different scales of the conventional physical testing pyramid (Figure 1). At the lower end of the testing pyramid, simulations take into account the physical mechanisms of damage and nonlinearity at the lamina level through a high-fidelity modelling approach. Modelling of the coupons is automated by means of Python scripting to allow for rapid generation of virtual allowables. Higher up the testing pyramid, at structural detail and panel level, it is not computationally efficient to model the composite structure in full detail. At this scale the emphasis is at global structural behaviour, for example during (post)buckling, and the performance of critical interfaces such as the welded skin-stiffener interface are of high importance. Separation of the welded skin-stiffener interface is modelled by the use of the Virtual Crack Closure Technique, both for damaged and pristine interfaces, allowing for large mesh sizes [3]. This allows for efficient modelling of skin-stiffener separation, even at the component level. Use is made of a virtual building block approach to ensure the validity of the methodology at each scale and corresponding critical failure mode.
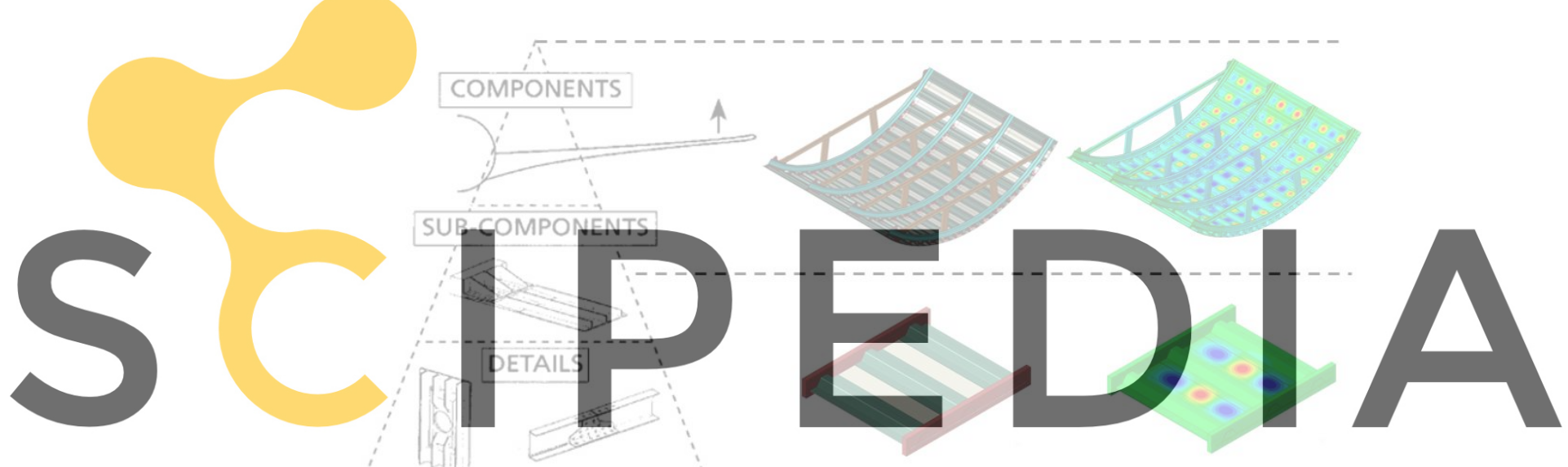

Register for free at https//WWEW Soripedia.com to download the version without the watermark

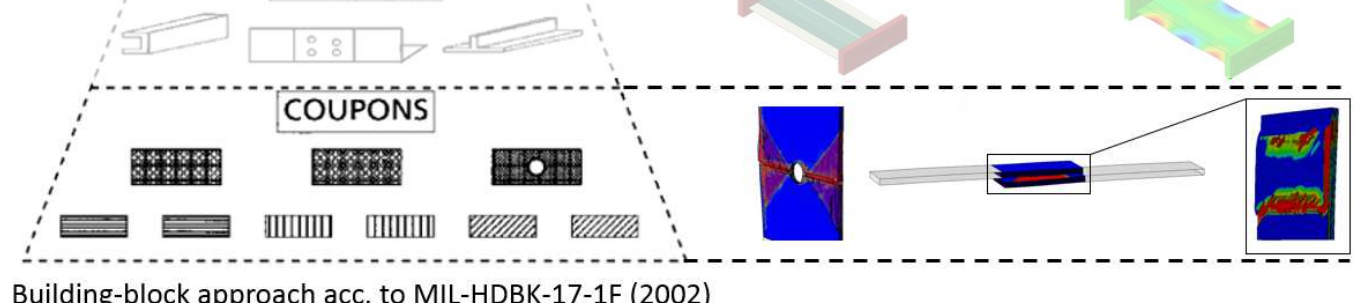

Figure 1: Virtual testing building block approach for thermoplastic composites

\section{NUMERICAL FRAMEWORK FOR VIRTUAL COUPON TESTING}

The numerical framework for Virtual Coupon Testing has been implemented through the use of the programming language Python using ABAQUS scripting commands to automatically generate coupon tests according to a given test matrix. This test matrix contains all user-defined input such as the test standard, specimen geometry and material data. The ABAQUS/Explicit finite element solver is selected for the coupon simulation, as it involves solving a highly nonlinear dynamic problem. This includes large displacements, non-linear material behavior including 
damage and complex contact interaction with damage and frictional behavior. The high-fidelity modelling approach and constitutive material model for thermoplastic composites is briefly explained in the next sections.

\subsection{High-fidelity modelling approach}

The modelling of the coupons follows a high-fidelity approach where each ply is discretized using a fiber aligned meshing technique [1] that behaves according to the constitutive material models briefly explained below. The plies are therefore modelled as separate parts and surface based general contact is used to facilitate the non-conformal meshes of each part as shown in Figure 2 . The boundary conditions are applied by means of a velocity amplitude profile imposed at the top and bottom surfaces in the case of standard uni-axial loading.

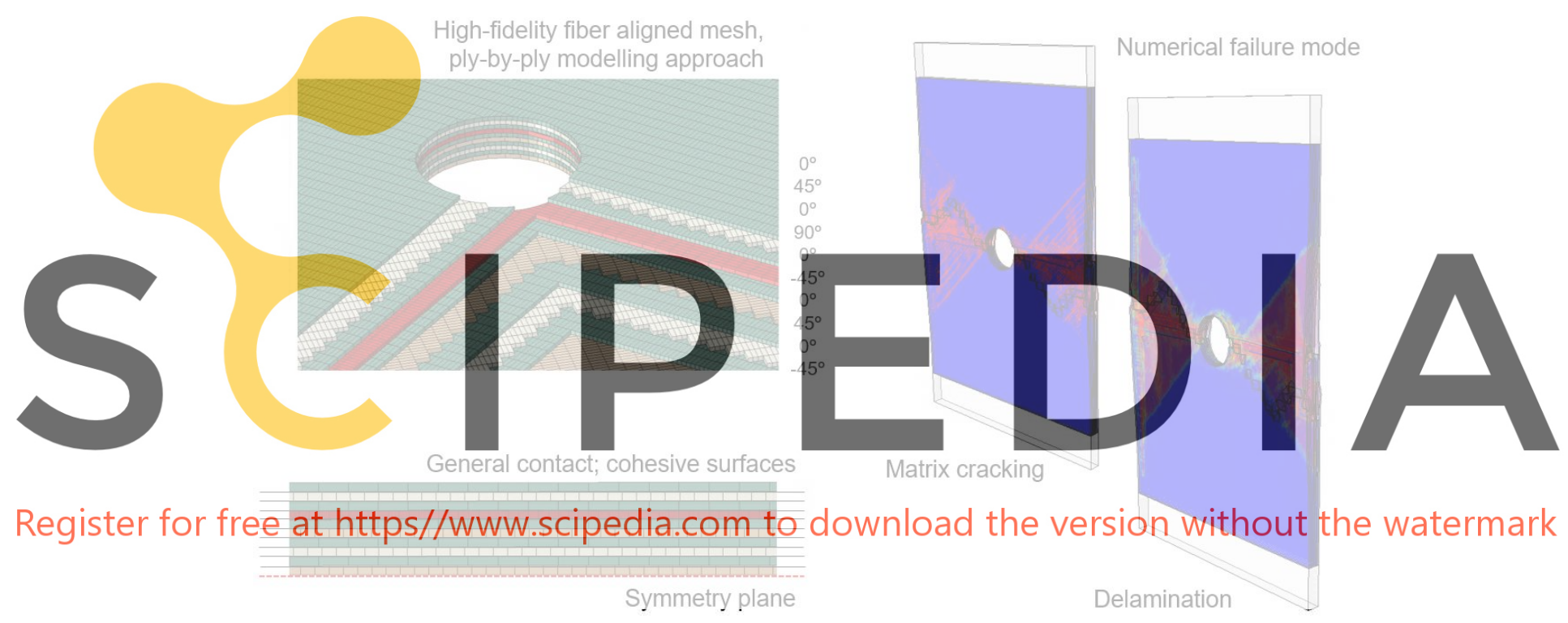

Figure 2: High-fidelity modelling approach and predicted open-hole failure mode

\subsection{Constitutive material model for thermoplastic composites}

The constitutive material model for thermoplastic composites consists is implemented in a user-defined subroutine "VUMAT" as a Continiuum Damage Model. The model takes into account three-dimensional stress states and is based on the deformation gradient to account for large shear deformations within the failure modes. This is important for accurate prediction of matrix dominated failure modes such as ply splits [2] as can be observed in the results shown in Figure 2. The laminate compliance tensor is affected by the damage variables in each orthotropic direction and is associated with the damage evolution laws for each failure mode. Exponential softening laws are used to ensure physically correct dissipation of fracture energy for each failure mode. Nonlinear elastic-plastic behavior of the matrix is modelled for both in-plane and out-plane shear response based on Ramberg-Osgood laws [1]. 
The interlaminar behavior is modelled by means of the build-in ABAQUS/Explicit general contact algorithm which takes care of the kinematics of surface contact, frictional and cohesive behavior. Damage initiation is predicted by using a quadratic nominal stress failure criteria. Once damage is initiated, the cohesive zone model allows for the correct dissipating of fracture energy corresponding to the specific mixed-mode opening mode. Frictional effects of the delaminations are also considered.

\subsection{Experimental validation}

Virtual test were performed and compared to a large variety of experimental results that include plain material strength, open-hole strength and single-lap shear joint strength of welded joints. Both the progressive failure mechanisms and the failure load are predicted with high accuracy with respect to the results obtained experimentally. Figure 2 shows the predicted matrix cracking and delaminations in an open-hole tensile test and Figure 3 demonstrates the progressive failure of a thermoplastic welded joint. The joint strength is highly influenced by the failure mechanisms of not only the welded interface but also the back-up plies which is important to consider when modelling simplifications are made at the structural level.
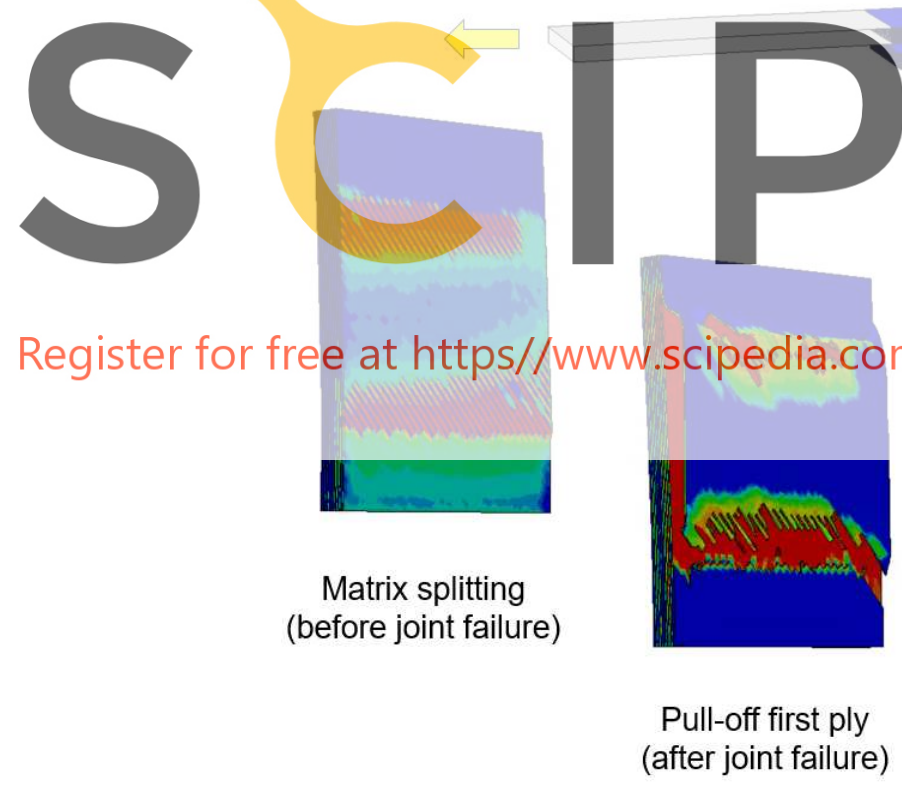

Welded single-lap shear joint loaded in tension

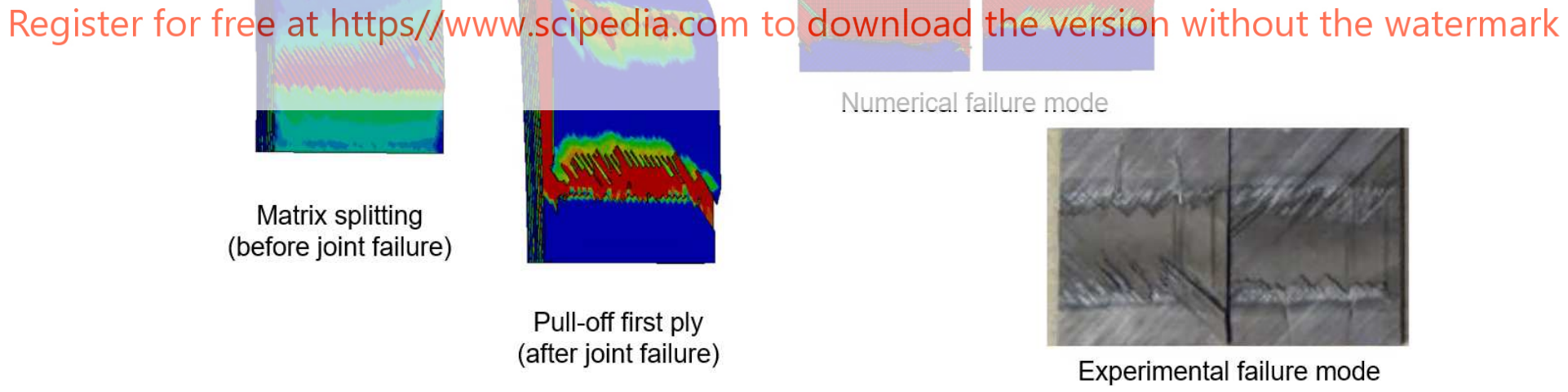

Figure 3: Virtual Coupon Test results of welded single-lap shear joint

\section{VIRTUAL STRUCTURAL TESTING}

The numerical framework for Virtual Structural Testing covers the structural detail, element and (sub)component level as shown in Figure 1. At this scale, the emphasis is on predicting the resulting (post)buckling strength due to compression loading and the failure behavior of the welded skin-stringer interface. The framework utilizes the ABAQUS/implicit dynamic solver 
for the finite element analysis and python coding is used to account for measured geometrical imperfections.

\subsection{Modelling approach to predict skin-stringer separation of welded joints}

Skin-stringer separation is modelled by means of the Virtual Crack Closure Technique (VCCT) in combination with the Benzeggagh Kenane criteria for mixed mode interface behavior. The use of VCCT allows for a coarser mesh compared to other interface methods, which is a major advantage when modelling large structures. The restriction of VCCT is its requirement for a pre-crack, which normally prevents it from being used for pristine structures. However, the unwelded areas on both sides of the welded joint can be modelled as a pre-crack to limit this restriction (Figure 4a). Furthermore, information on the material strength of the skin and stringer may be taken from predictions at the lower scale in order to avoid computationally expensive analyses at the structural level.
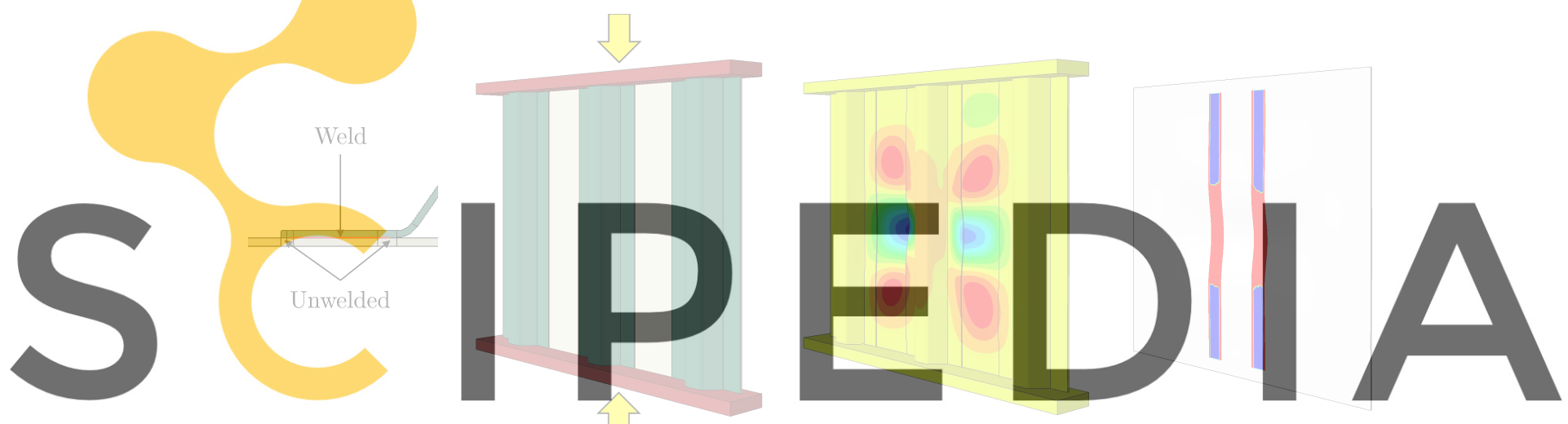

Register for free at https//www.scipedia.com to download the version witklout the watermark

Figure 4: (a) Modelling strategy for welded interface; (b) Multi-stringer panel under compression; (c) Opening of skin-stringer interface; (d) Interface failure.

\subsection{Design support of the Next Generation Thermoplastic Multifunctional Fuselage}

The new numerical framework for Virtual Testing may be used to form the basic buildingblocks early on the design process when new materials and design concepts are used. In the case of the Next Generation Thermoplastic Multifunctional Fuselage, this involves both thermoplastic composites and welded joints. Developing the simulation strategy to virtual test structural (sub)components such as compression panels of a new product follows both a top-down and bottom-up strategy. At the top level, the loading conditions and design requirements are defined and simulations at the component level provide input about the expected mechanical behavior at the (sub)structural level. For the case of the new fuselage, which consists of a lightweight thermoplastic composite stiffened structure that is joined with welding, the main concerns are related to the strength of these joints during complex loading conditions such as (post)buckling and the capability of the structure to be tolerant to damages at these interfaces. Knowledge from the bottom-up material design allowables and failure modes is therefore linked with the 
top-down requirements to design structural detail and elements that will cover these loading and damage scenarios. Examples of virtual structural tests that may be designed are singleand multi-stiffener compression panels that could also include damages at the welded interface based on impact scenarios. Example of these models are shown in Figure 1 and 4. The aim of this strategy is to verify and validate the analysis methods at the lower scale and predict the effect of damage at the higher scale under various loading or damage conditions, as physical testing every possible configuration is too expensive. Through this strategy the efficiency of new joining techniques and material behavior can be explored while at the same time it may reduce the risks of introducing new design concepts early on in the design phase. This rapidly increases the design knowledge and allows for a smarter design of the physical tests.

\section{CONCLUSIONS}

It is demonstrated that the new numerical framework for Virtual Testing can support accurate predictions of the failure of thermoplastic composites and welded joints through the different scales of the physical testing pyramid. It can provide the basic design building-blocks when availability of experimental data and design knowledge is limited and allows for a smarter design of the physical tests later on in the design process.

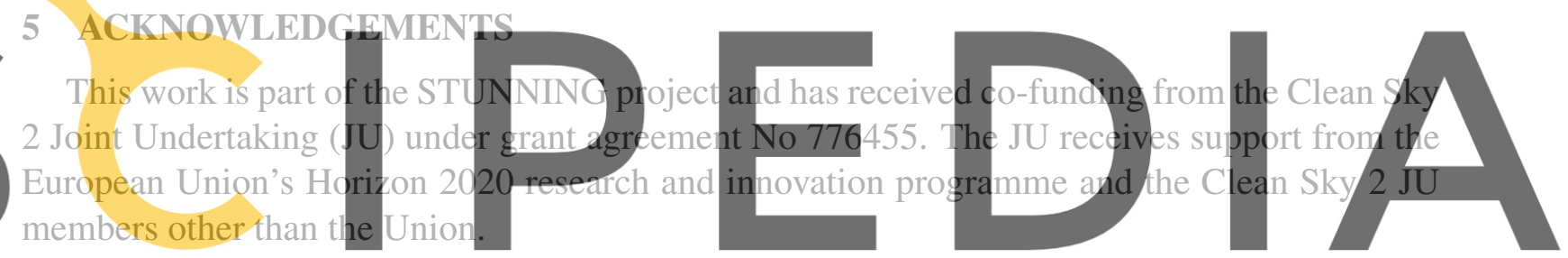

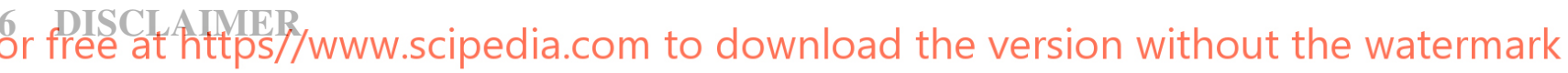

The results, opinions, conclusions, etc. presented in this work are those of the author(s) only

and do not necessarily represent the position of the JU; the JU is not responsible for any use made of the information contained herein.

\section{REFERENCES}

[1] Falcó, O., Ávila, R.L., Tijs, B. and Lopes, C.S. Modelling and simulation methodology for unidirectional composite laminates in a Virtual Test Lab framework. Composite Structures (2018) 190:137-159.

[2] Tijs, B.H.A.H., Turon, A. and Bisagni, C. The importance of accounting for large shear deformation on modelling matrix failure of thermoplastic and thermoset composites. Proceedings of the 7th ECCOMAS Thematic Conference on the Mechanical Response of Composites (COMPOSITES 2019), Girona, Spain, Sept. 18-20 (2019).

[3] van Dooren, K.S., Labans, E., Tijs, B.H.A.H., Waleson, J.E.A., and Bisagni, C. Analysis and testing of a thermoplastic composite stiffened panel under compression. Proceedings of the 22nd International Conference on Composite Materials (ICCM22), Melbourne, AU, Aug. 11-16 (2019). 